\title{
Design and control of nonlinear mechanical systems for minimum time
}

\author{
J.B. Cardoso ${ }^{\mathrm{a}, *}$, P.P. Moita ${ }^{\mathrm{b}}$ and A.J. Valido ${ }^{\mathrm{b}}$ \\ ${ }^{a}$ Instituto Superior Técnico, Departamento de Engenharia Mecânica, Av. Rovisco Pais, 1049-001 Lisboa, Portugal \\ ${ }^{\mathrm{b}}$ Escola Superior de Tecnologia de Setúbal, Campus do IPS, 2914-508 Setúbal, Portugal
}

Received 2007

Revised 2007

\begin{abstract}
This paper presents an integrated methodology for optimal design and control of nonlinear flexible mechanical systems, including minimum time problems. This formulation is implemented in an optimum design code and it is applied to the nonlinear behavior dynamic response. Damping and stiffness characteristics plus control driven forces are considered as decision variables. A conceptual separation between time variant and time invariant design parameters is presented, this way including the design space into the control space and considering the design variables as control variables not depending on time. By using time integrals through all the derivations, design and control problems are unified. In the optimization process we can use both types of variables simultaneously or by interdependent levels. For treating minimum time problems, a unit time interval is mapped onto the original time interval, then treating equally time variant and time invariant problems. The dynamic response and its sensitivity are discretized via space-time finite elements, and may be integrated either by at-once integration or step-by-step. Adjoint system approach is used to calculate the sensitivities.
\end{abstract}

Keywords: Minimum time design, nonlinear systems, dynamic response, space-time finite elements

\section{Introduction}

Structures and flexible mechanical systems, by one hand, as well as optimal design and optimal control, by another hand, have been traditionally treated with separated formulations. As the theory and methods of nonlinear structural analysis have progressed, there is no distinction between flexible mechanical systems and structures. Also, in the last 15 years there has been the integration of optimal design and optimal control problems [1-4].

Numerical methods for the solution of optimum control problems are divided into two major approaches: methods based on the Pontryagin's maximum principle and direct methods. The former ones need considerable preparatory work to derive the adjoint differential equations and are very sensitive to the initial estimate of the control variables and to the integration of adjoint differential equations. Direct methods transform an infinite dimensional optimal control problem into a finite dimension optimization problem [5,6].

This paper presents an integrated methodology for optimal design and control of nonlinear flexible mechanical systems. In order to implement it, one uses: (i) a nonlinear structural finite element technique to model large displacements, referring all the quantities to an inertial frame and using stress and strain measures that are invariant with the rigid body motion [7]; (ii) a conceptual separation between time variant and time invariant design parameters, this way including the design space into the control space and considering the design variables as control variables not depending on time. By using time integrals through all the derivations, the design and control problems are

${ }^{*}$ Corresponding author. E-mail: barradas@ist.utl.pt. 
unified. In the optimization process we can use both types of variables simultaneously or by interdependent levels. Both types are designated here as design variables.

Minimum time problems are considered. Time variation is treated by mapping a fixed time domain onto the original time domain, extending the idea of fixed reference volume [8] to the time domain. This fashion, the time domain turns free of design variables, then the jacobian of the transformation being dependent on them. The total time interval is therefore considered a new state variable.

A bound formulation is applied to handle the multicriteria problems. To model the systems, a space-time finite element discretization is utilized and the solution of the dynamic equations is obtained either by at-once method or step-by-step.

The aim of the Design Sensitivity Analysis (DSA) is the calculation of the performance measure gradients with respect to the design variables. It represents an important tool for design improvement and it is a necessary stage within the optimization process. A general overview of the DSA problems and methods of nonlinear structural mechanics is given elsewhere [9]. Basically, the methods may be classified as direct differential methods and adjoint system methods. Both are applied in this work. For minimum time problems there is no way of checking the sensitivities by finite differences comparison. In this case one needs to compare them with closed form solutions.

The response analysis and corresponding DSA are implemented in the interactive optimal design code OPTIMISE in order to use optimality criteria or nonlinear programming optimization runs.

\section{Response analysis}

The virtual work dynamic equilibrium equation of the system at the time $t$ is given as

$$
\delta W=\int\left(\rho^{t} \ddot{u} \cdot \delta^{t} u+{ }^{t} S \cdot \delta^{t} \varepsilon-{ }^{t} f \cdot \delta^{t} u\right)^{0} d V-\int{ }^{t} T \cdot \delta^{t} u{ }^{0} d \Gamma
$$

where all the quantities are referred to the undeformed configuration, $\delta$ represents variation of the state fields, ' ' refers to the standard tensor product, upper dot '? refers to the material time derivative, $\rho$ is the mass density at time $t=0,{ }^{t} u$ is the displacement, ${ }^{t} S$ is the second-Piola stress measure, ${ }^{t} \varepsilon$ is the Green strain tensor, ${ }^{t} f$ is the body force per unit volume, ${ }^{t} T$ is the surface traction, ${ }^{0} V$ is the undeformed volume of the body, and ${ }^{0} \Gamma$ is the surface of the undeformed body.

A space-time finite element model is used to discretize the dynamic analysis response. After space discretization we have the governing matrix equation as

$$
M^{t} \ddot{U}+{ }^{t} C_{S}^{t} \dot{U}+{ }^{t} K_{S}^{t} U={ }^{t} P
$$

where $M$ is the mass matrix, ${ }^{t} C_{S} \equiv{ }^{t} C_{S}\left({ }^{t} \dot{U}\right),{ }^{t} K_{S} \equiv{ }^{t} K_{S}\left({ }^{t} U\right)$ are respectively the damping and stiffness matrices, ${ }^{t} P$ is the loading vector and ${ }^{t} U,{ }^{t} \dot{U},{ }^{t} \ddot{U}$ are respectively the displacement, velocity and acceleration vectors, all the quantities defined at time $t$.

For temporal modeling, finite elements of dimension $\Delta t$ were considered, selecting hermitean cubic elements to model the displacements and quadratic lagrangean elements to model the loading, extending the algorithm given in [7] to the case of nonlinear systems. By taking the time derivative of the Eq. (2) by one hand, and by another hand its integration once and then twice with average values of stiffness and damping in $\Delta t$ given as

$$
\bar{K} \equiv{ }^{t+\Delta t / 2} K_{S}\left({ }^{t} U+{ }^{t} \dot{U} \Delta t / 2\right), \bar{C} \equiv{ }^{t+\Delta t / 2} C_{S}\left({ }^{t} \dot{U}+{ }^{t} \ddot{U} \Delta t / 2\right)
$$

one obtains four equations that combine to give the dynamic finite time-element equation as

$$
D_{S}^{e} z^{e}=P^{e}
$$

where

$$
D_{S}^{e}=\left[\begin{array}{llllll}
{ }^{t} K_{S} & { }^{t} C_{S} & M & 0_{n x n} & 0_{n x n} & 0_{n x n} \\
{ }^{t} D_{S_{11}}{ }^{t} D_{S_{12}} & 0_{n x n}{ }^{t+\Delta t} D_{S_{11}}{ }^{t+\Delta t} D_{S_{12}} & 0_{n x n} \\
{ }^{t} D_{S_{21}} & { }^{t} D_{S_{22}} & 0_{n x n} & { }^{t+\Delta t} D_{S_{21}}{ }^{t+\Delta t} D_{S_{22}} & 0_{n x n}
\end{array}\right], \quad P^{e}=\left\{\begin{array}{l}
{ }^{t} P \\
P_{1} \\
P_{2}
\end{array}\right\}
$$

and 


$$
z^{e}=\left({ }^{t} z,{ }^{t+\Delta t} z\right), \quad{ }^{t} z=\left({ }^{t} U,{ }^{t} \dot{U},{ }^{t} \ddot{U}\right)
$$

In Eq. (5) $n$ stands for number of space degrees-of-freedom and ${ }^{t} D_{S_{j k}}$ are functions of $\bar{K}, \bar{C}, M$. The Eq. (4) may be solved step-by-step, i.e., element-by-element in time, or assembled to be solved at-once. In this case, we have to assemble for a total time interval $T$ discretized in $\mathrm{N}$ time nodes, resulting in the dynamic equation

$$
D_{S} z=P
$$

where $2 n$ time boundary conditions are imposed by transferring the corresponding columns of the assembled matrix $D_{S}$ to the right-hand side of Eq. (7) after multiplying by the vector $U_{c}$ of those conditions, resulting the equation

$$
\hat{K}_{S} \hat{U}=\hat{P}, \quad \hat{P}=P-D_{c} U_{c}
$$

The Eq. (8) is a nonlinear equation where $\hat{K}_{S}$ is a nonsymmetrical matrix dependent on the response. Therefore, the Eq. (8) has to be solved iteratively.

\section{Design sensitivity analysis}

Consider now a general performance measure defined in the time interval $[0, T]$ as

$$
\Psi=\int G\left({ }^{t} z, b, t\right) d t
$$

where ${ }^{t} b$ is the vector of design and control variables and the other quantities were defined in Eqs (1) and (6). The external forces ${ }^{t} P$ generally belong to the design vector. For minimum time problems consider also a terminal condition

$$
{ }^{T} \Phi\left({ }^{T} z,{ }^{T} b, T\right)=0
$$

The design sensitivity analysis problem is to derive the total design variation of the measure in Eq. (9) with respect to the design ${ }^{t} b$, for the system represented by the equation of motion, Eq. (8), and subject to the terminal condition of the Eq. (10).

\subsection{The adjoint method of design sensitivity analysis for minimum time problems}

The total design variation for the performance measure of Eq. (9) is

$$
\bar{\delta} \Psi=\overline{\bar{\delta}} \Psi+\tilde{\delta} \Psi+\Psi, t \bar{\delta} T
$$

where $\overline{\bar{\delta}} \Psi$ and $\tilde{\delta} \Psi$ represent respectively the explicit and implicit (state dependent) design variations. Although the total time $T$ is also considered as a state variable, it is here treated separately of the other state variables.

In order to formulate design sensitivity analysis one is going to use the adjoint system method. For that, replace the arbitrary variation of state fields by adjoint fields into the virtual work equation as

$$
W^{a}=\left(\hat{K}_{S} \hat{U}-\hat{P}\right) \cdot \hat{U}^{a}=0
$$

and define an extended 'action' function

$$
A=\Psi-W^{a}
$$

The basic idea of introducing an adjoint system is to replace the implicit design variations of the state fields by explicit design variations and adjoint fields, then determining these adjoint fields by vanishing the implicit design variation of the 'action' function $A[4]$ as

$$
\tilde{\delta} A=0
$$

then the total design variation of $\Psi$ can be written as

$$
\bar{\delta} \Psi=\overline{\bar{\delta}} A+\Psi{ }_{t} \bar{\delta} T
$$




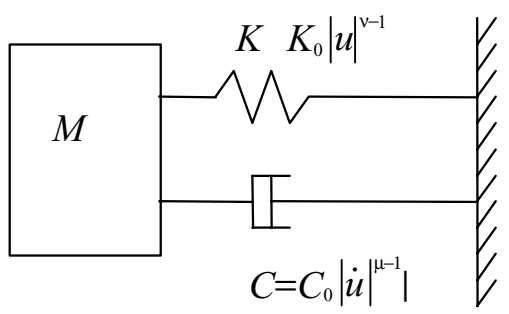

Fig. 1. Impact absorber.

The total time variation may be now determined by imposing the terminal condition of the Eq. (10) after any design variation, i.e.

$$
\bar{\delta}^{T} \Phi=\overline{\bar{\delta}}^{T} \Phi+\tilde{\delta}^{T} \Phi+{ }^{T} \Phi,{ }_{,} \bar{\delta} T=0
$$

or

$$
\bar{\delta} T=-\left(\overline{\bar{\delta}}^{T} \Phi+\tilde{\delta}^{T} \Phi\right) /{ }^{T} \Phi, t
$$

to substitute into the Eq. (15) and get the total design variation of the measure of interest.

\subsection{Design sensitivity analysis modeling}

In order to solve the design sensitivity analysis problem, the sensitivities are firstly performed at the element level and then the sensitivity equations are assembled. The explicit design variation of the vector of element forces of Eq. (4) is

$$
\overline{\bar{\delta}} R^{e}=\overline{\bar{\delta}} P^{e}-\overline{\bar{\delta}} F^{e}, \quad F^{e}=D_{S}^{e} z^{e}
$$

and the implicit design variation of the internal forces gives

$$
\tilde{\delta} F^{e}=\tilde{\delta} D_{S}^{e} z^{e}+D_{S}^{e} \tilde{\delta} z^{e}=D^{e} \tilde{\delta} z^{e}
$$

where

$$
D^{e}=\left[\begin{array}{llllll}
{ }^{t} K & { }^{t} C & M & 0_{n x n} & 0_{n x n} & 0_{n x n} \\
{ }^{t} D_{11}{ }^{t} D_{12}{ }^{t} D_{13} & { }^{t+\Delta t} D_{11}{ }^{t+\Delta t} D_{12} & 0_{n x n} \\
{ }^{t} D_{21}{ }^{t} D_{22} & { }^{t} D_{23}{ }^{t+\Delta t} D_{21} & { }^{t+\Delta t} D_{22} & 0_{n x n}
\end{array}\right]
$$

with

$$
{ }^{t} K={ }^{t} K_{S}+{ }^{t} K_{S, U}{ }^{t} U, \quad{ }^{t} C={ }^{t} C_{S}+{ }^{t} C_{S, \dot{U}}{ }^{t} \dot{U}
$$

Sensitivities of Eq. (18) and the element dynamic matrix of Eq. (20) are assembled and again imposed the time boundary conditions resulting respectively $\overline{\bar{\delta}} \hat{R}$ and $\hat{K}$.

Now, the application of the Eq. (14) to the Eq. (13) gives the adjoint system equilibrium

$$
\hat{K}^{T} \hat{U}^{a}=(\Psi, \hat{U})^{T}
$$

Thus, the total design variation of Eq. (15) is

$$
\bar{\delta} \Psi=\overline{\bar{\delta}} \Psi+\hat{U}^{a} \cdot \overline{\bar{\delta}} \hat{R}
$$


U

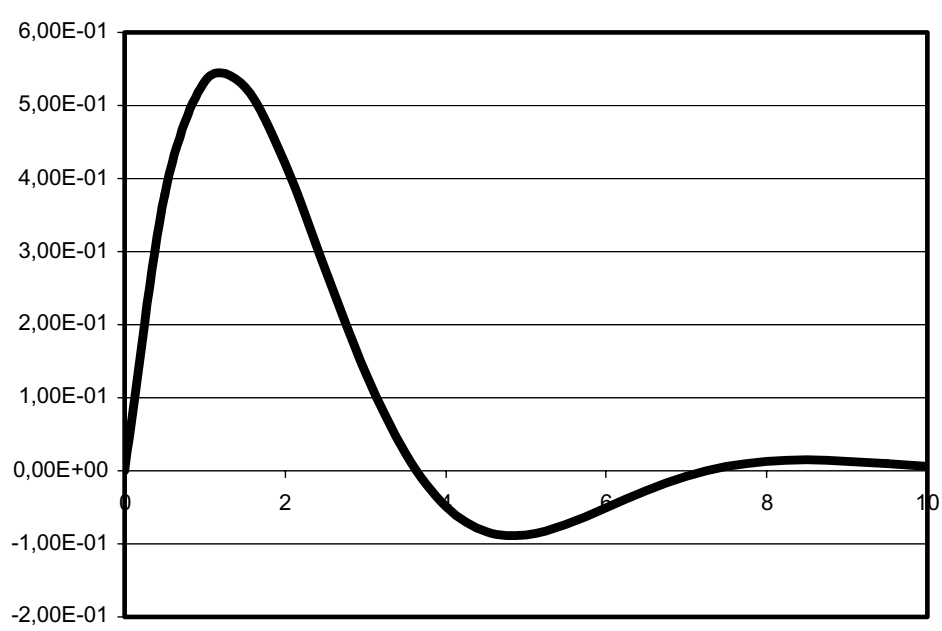

Fig. 2. Starting design displacement [m].

V

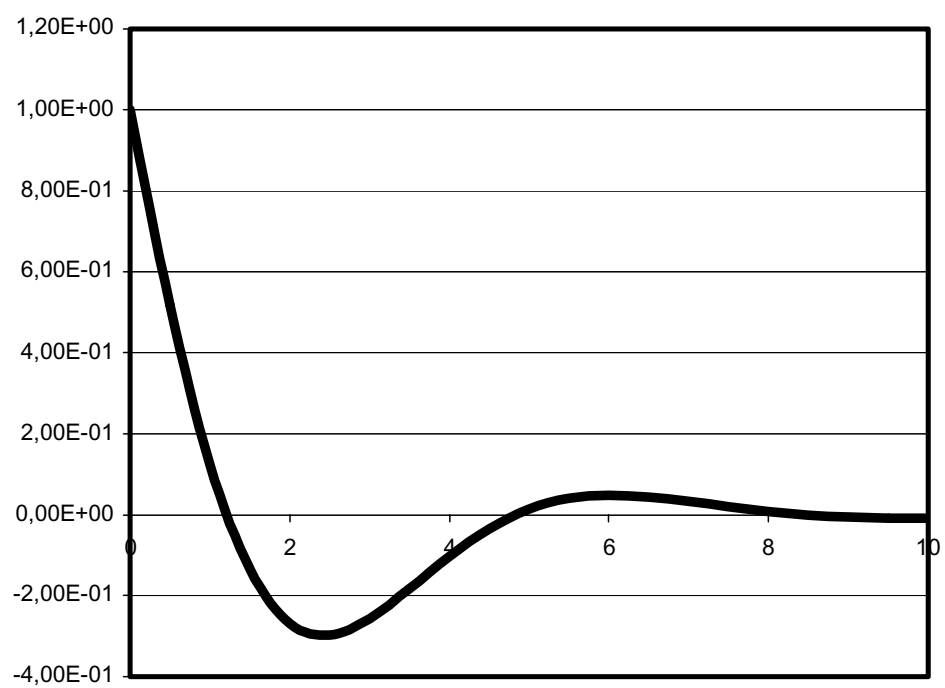

Fig. 3. Starting design velocity $[\mathrm{m} / \mathrm{s}]$.

\section{Optimum design problem}

For minimum time problems, the merit measure is the total time interval, expressed as

$$
\Psi_{0} \equiv T=\int d t
$$

Because the final time is an unknown quantity, a difficulty arises for the time discretization. Since the problem domain varies as the optimization iterates progress, a fixed unit time interval is mapped onto the original time interval $T$, and the original time $t$ is transformed to the non-dimensional time $\tau=t / T$ such that we have

$$
d / d t=T^{-1} d / d \tau, \quad d^{2} / d t^{2}=T^{-2} d^{2} / d \tau^{2}
$$

All the performance measures are transformed accordingly, that is 
a

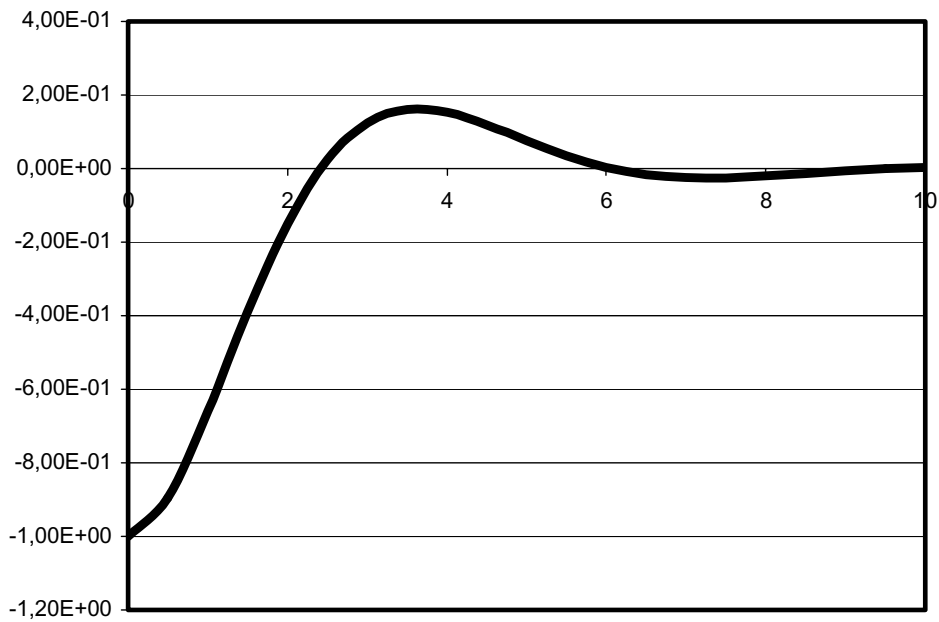

Fig. 4. Starting design acceleration $\left[\mathrm{m} / \mathrm{s}^{2}\right]$.

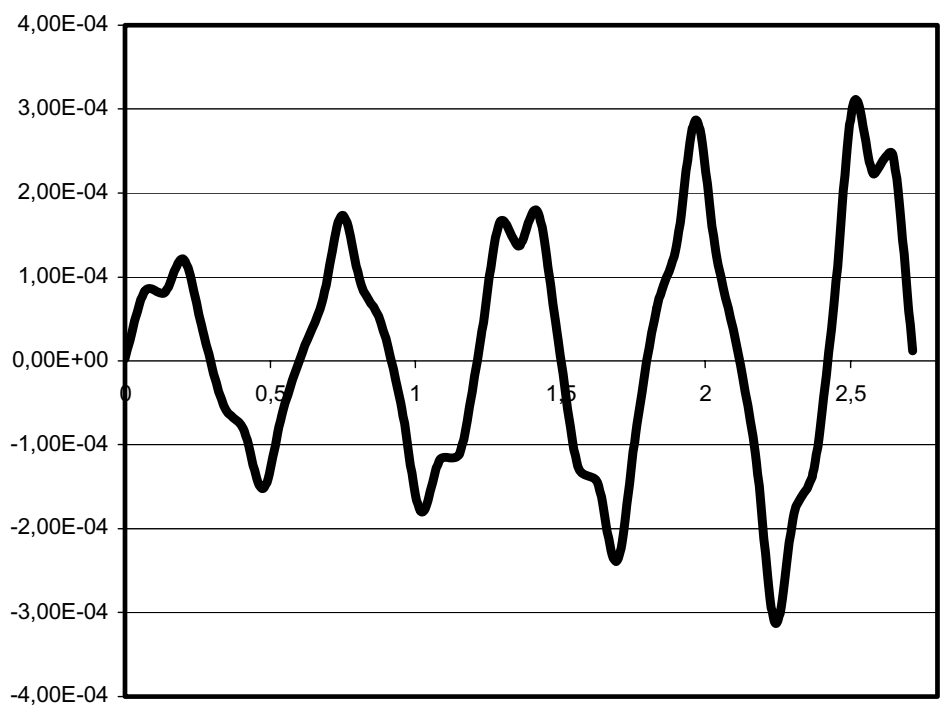

Fig. 5. Optimal multicriterium control force $[\mathrm{N}]$.

$$
\bar{\Psi}_{0}=\int T d \tau, \quad \bar{\Psi}=\int \bar{G}\left({ }^{\tau} \bar{z},{ }^{\tau} \bar{b}, \tau\right) T d \tau, \quad{ }^{1} \bar{\Phi}\left({ }^{1} \bar{z},{ }^{1} \bar{b}, T\right)=0
$$

All the analysis and design sensitivity analysis may be ran for a fixed unit time interval with mass, damping, velocities and accelerations transformed as

$$
m=T^{-2} M, \quad c=T^{-1} C, \quad U^{\prime}=T \dot{U}, \quad U^{\prime \prime}=T^{2} \ddot{U}, \quad ' \equiv d / d \tau
$$

In order to solve the multicriterium problems, a bound formulation is used. A new fictitious design variable $\beta$ is introduced which is also a new objective and an upper bound for the original objectives. This way, the optimization problem is formulated as

$$
\min \beta, \quad \text { s.t. } \quad \Psi_{0 k} \leqslant \beta, \quad\left(k=1,2, \ldots, m_{0}\right) ; \quad \Psi_{j} \leqslant 0,(j=1,2, \ldots, m) ; \quad{ }^{T} \Phi=0
$$


u

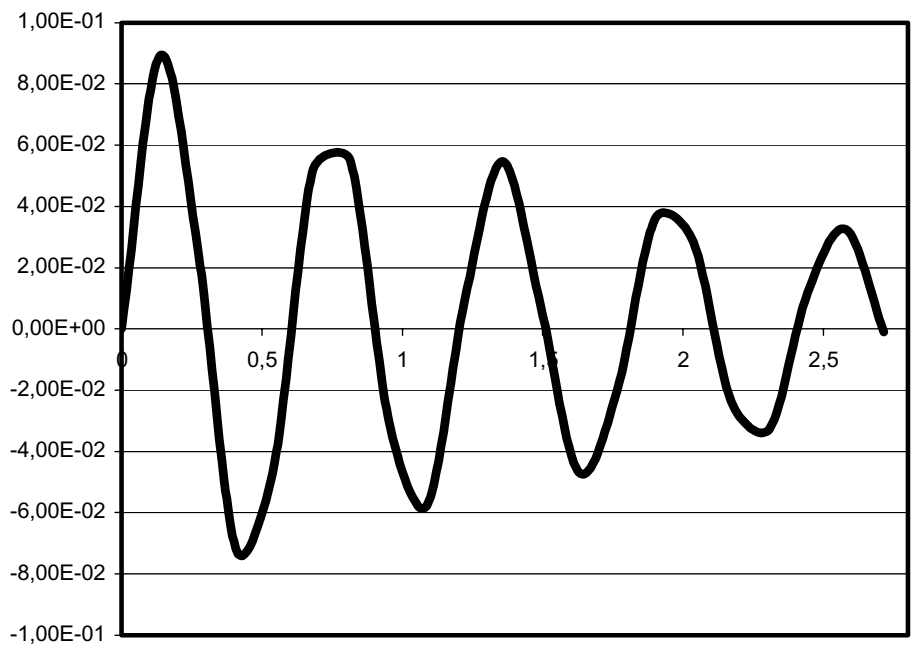

Fig. 6. Optimal multicriterium design displacement [m].

V

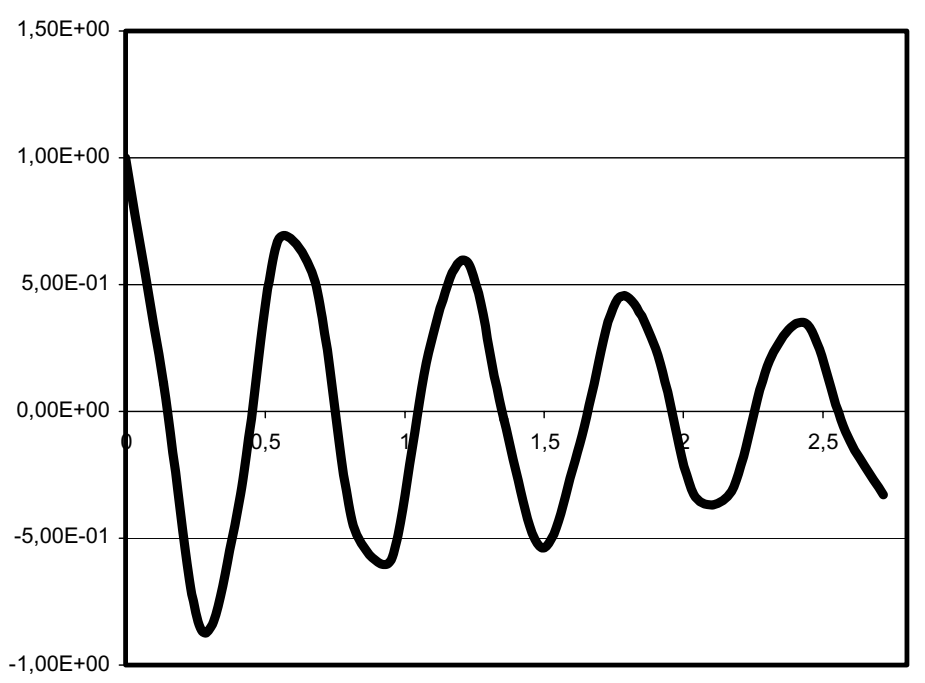

Fig. 7. Optimal multicriterium design velocity $[\mathrm{m} / \mathrm{s}]$.

where $\Psi_{0 k}$ are the original objectives (now new constraints) and $\Psi_{j}$ are the original constraints. The formulation of Eq. (28) may be considered a general formulation for multicriterium as well as for unicriterium problems.

\section{Numerical example}

\subsection{Impact absorber for minimum time and mean square velocity}

The impact absorber of the Fig. 1, with $\nu=\mu=1$, is optimized with respect to the spring and damping coefficients, and to the driven control force $\left[{ }^{t} P, 0 \leqslant t \leqslant T\right]$, in order to minimize two merits: the total time $T$ and the mean square velocity $E=\int \dot{u}^{2} d t$. The mean displacement constraint $\left|u_{m}\right| \leqslant 0.01 \mathrm{~m}$ and the terminal 
a

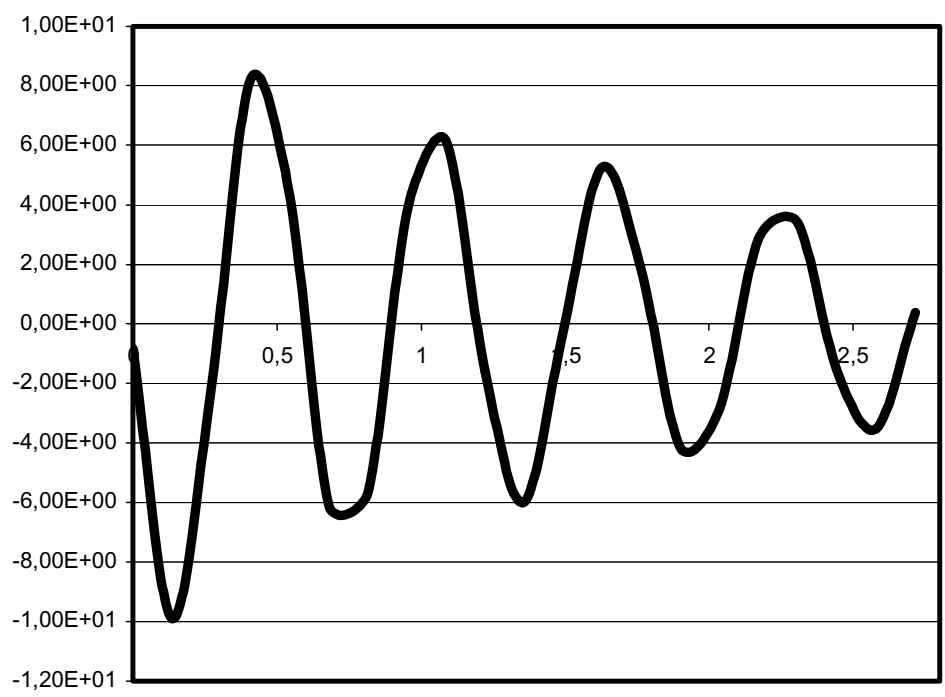

Fig. 8. Optimal multicriterium design acceler. $\left[\mathrm{m} / \mathrm{s}^{2}\right]$.

displacement constraint ${ }^{T} u=0$ are imposed. The system impacts with the velocity $\dot{u}=1 \mathrm{~m} / \mathrm{s}$ at $t=0$. The starting design in this example is $b \equiv\left[\beta=1 ; K_{0}=1 \mathrm{~N} / \mathrm{m} ; C_{0}=1 \mathrm{Ns} / \mathrm{m} ;{ }^{t} P=0,(0 \leqslant t \leqslant T)\right]$, where $T=10 \mathrm{~s}$, with which one obtains $E=0.499 \mathrm{~m}^{2} / \mathrm{s}$. The starting responses of displacement, velocity and acceleration are given in Figs 2, 3 and 4, respectively.

The optimization was performed by levels, firstly with respect to $K_{0}$ and $C_{0}$ and then with respect to the driven control force ${ }^{t} P$. The optimum design values were obtained as $K_{0}=109.96 \mathrm{~N} / \mathrm{m}$ and $C_{0}=0.84043 \mathrm{Ns} / \mathrm{m}$ and the optimal control force as represented in Fig. 5. The final optimum values of the objectives were obtained as $T=$ $2.715 \mathrm{~s}$ and $E=0.52082 \mathrm{~m}^{2} / \mathrm{s}$ with a maximum violation equal to $3.75352 \mathrm{e}-4$.

The displacement, velocity and acceleration responses for the optimum design are given in the Figs 6, 7 and 8 .

\section{Concluding remarks}

A design sensitivity analysis and an optimal design formulation have been developed to perform minimum time problems. By extending the spatial domain to the space-time domain, the design space is included in the control space. Thus, one can unify in one single formulation the problems of optimum design and optimal control, treating the design variables as control variables that do not change with time.

Using at-once integration of the equations of motion, and its sensitivities, the adjoint structure method can be used with all of its advantages and without its main disadvantage in dynamics: the necessity of backwards integration of the adjoint system. So, the needing of backwards integration with its major drawback, the memorization of the response history, is not associated with path-dependent problems, as it is frequently assumed, but it is associated with the fact of using step-by-step integration. It is also possible and easy to apply temporal boundary conditions at any point in time, not necessarily at the start of the integration, as in the step-by-step integration method.

For minimum time control problems, since the domain varies as the optimization proceeds, a fixed reference unit time interval is mapped onto the original time interval and total time is considered as a state variable. After the transformation of all the responses and optimization measures to the new unit time domain, the minimum time problem may be treated as a fixed time interval problem. This way, fixed time interval problems and varying time interval problems can be treated in a unified fashion. 


\section{References}

[1] M. Salama, J. Garba, L. Demsetz and F. Udwadia, Simultaneous Optimization of Controlled Structures, Computational Mechanics Mech 3 (1988), 275-282.

[2] C.H. Tseng and J.S. Arora, Optimum Design of Systems for Dynamics and Controls using Sequential Quadratic Programming, AIAA Journal 27(12) (1989), 1793-1800.

[3] J.S. Arora and T.C. Lin, A Study of Augmented Lagrangean Methods for Simultaneous Optimization of Controls and Structures, Fourth AIAA/Air Force/NASA/OAI Symposium, Cleveland, Ohio, USA, 1992.

[4] J.B. Cardoso and J.S. Arora, Design Sensitivity Analysis of Nonlinear Dynamic Response of Structural and Mechanical Systems, Structural Optimization 4 (1992), 37-46.

[5] J.H. Heilig and J. McPhee, Determination of Minimum-Time Maneuvers for a Robotic Manipulator using Numerical Optimization Methods, Mechanical Structures and Machines 27(2) (1999), 185-201.

[6] E. Polak, On the use of Consistent Approximations in the Solution of Semi-infinite Optimization and Optimal Control Problems, Mathematical Programming 62 (1993), 385-415.

[7] J.B. Cardoso, L. Sousa, J. Castro and A. Valido, Optimal Design of Nonlinear Structures and Mechanical Systems, Engineering Optimization 29 (1997), 277-291.

[8] J.B. Cardoso and J.S. Arora, Variational Method for Design Sensitivity Analysis in Nonlinear Structural Mechanics, AIAA Journal 26(5) (1988), 595-603.

[9] M. Kleiber, H. Antunez, T. Hien and P. Kowalczyk, Parameter Sensitivity in Nonlinear Mechanics, John Wiley \& Sons, 1997. 

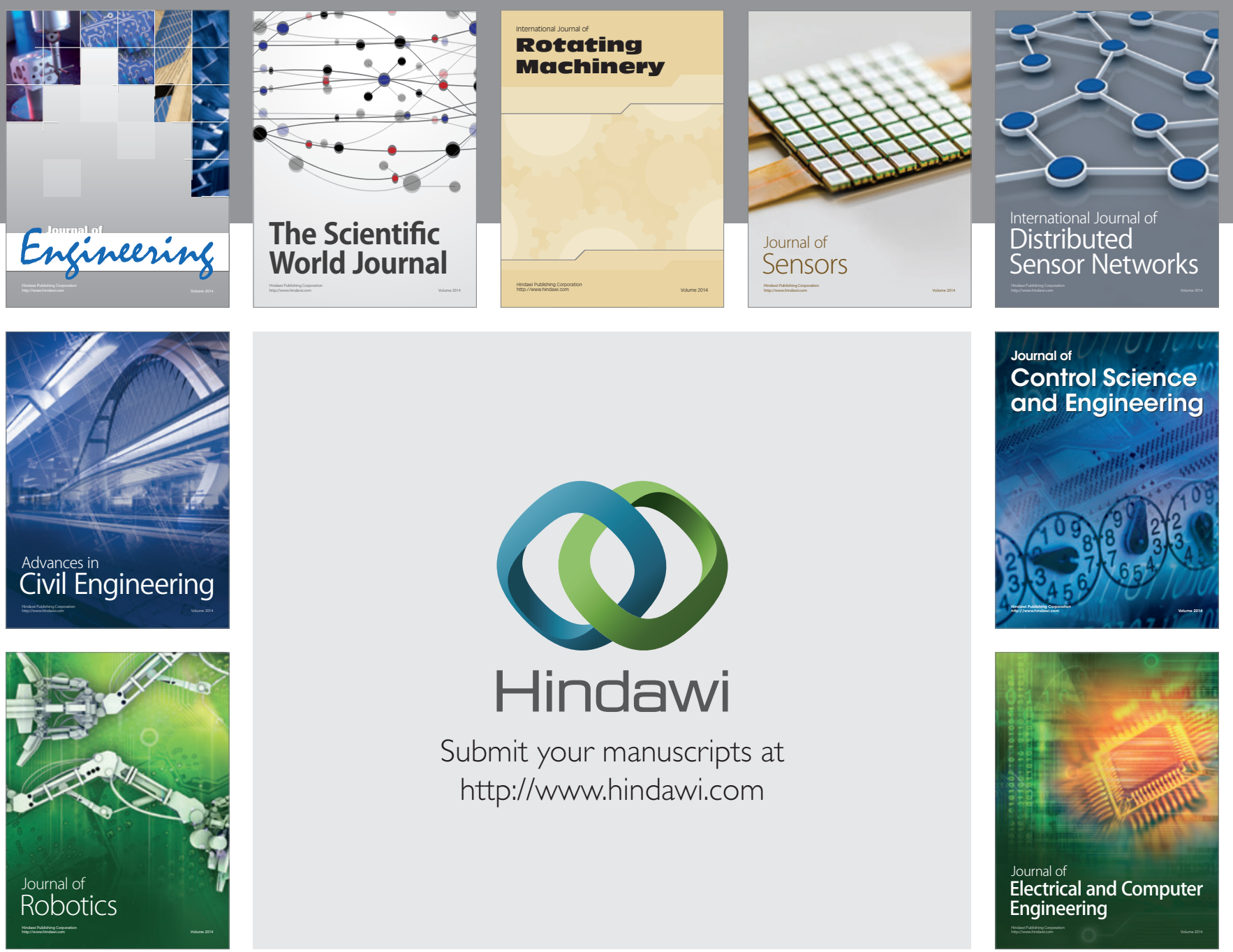

Submit your manuscripts at

http://www.hindawi.com
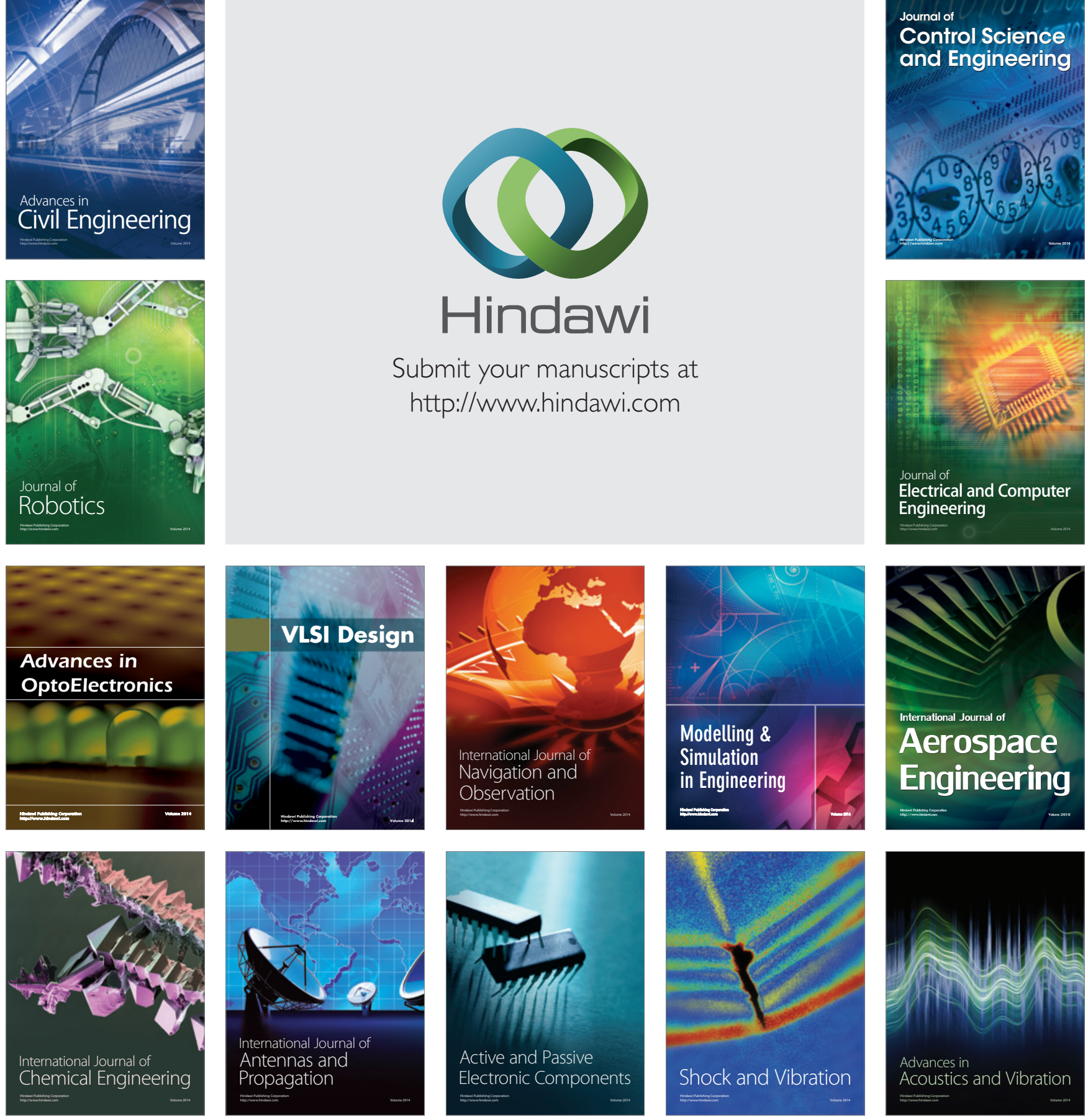